\title{
DISTRIBUTIONS OF CHERRIES FOR TWO MODELS OF TREES
}

\author{
ANDY McKENZIE and MIKE STEEL \\ Biomathematics Research Centre \\ University of Canterbury \\ Private Bag 4800 \\ Christchurch, New Zealand
}

No. 177

May, 1999 


\title{
Distributions of cherries for two models of trees
}

\author{
May 6, 1999
}

\author{
Andy McKenzie and Mike Steel \\ Biomathematics Research Centre \\ University of Canterbury \\ Christchurch, New Zealand
}

This research was supported by the New Zealand Marsden Fund (UOC-MIS-003)

\begin{abstract}
Null models for generating binary phylogenetic trees are useful for testing evolutionary hypotheses and reconstructing phylogenies. We consider two such null models - the Yule and uniform models - and in particular the induced distribution they generate on the number $C_{n}$ of cherries in the tree, where a cherry is a pair of leaves each of which is adjacent to a common ancestor. By realizing the process of cherry formation in these two models by extended Polya urn models we show that $C_{n}$ follows a normal distribution. We also give exact formulas for the mean and standard deviation of the $C_{n}$ in these two models. This allows simple statistical tests for the Yule and uniform null hypotheses.
\end{abstract}

\section{Introduction}

Phylogenetic trees are widely used in biology to represent evolutionary relationships between species. The shapes and branch lengths of such trees convey useful information regarding, for example, the rate of species formation and the occurrence of mass extinctions [1]. An unresolved debate concerns the relative importance of adaptive and stochastic factors in the process of lineage diversification. Adaptive factors are characteristics of a taxon thought to be responsible for the particular pattern of species survival or extinction observed in that taxon. Stochastic factors are those factors, random in appearance, that independently and uniformly effect the formation and extinction of all species in a tree.

Early stochastic modeling on phylogenetic trees concentrated on a qualitative comparison between actual phylogenetic trees and those produced in simulation studies [2,3]. While suggestive, such work suffered from the lack of an explicit measure of the degree of similarity between actual and simulated trees. Later quantitative work, based on simple stochastic models of the process of species formation and extinction, introduced measures of similarity based on the frequency of tree topologies $[4,5,6]$ and tree symmetry $[7,8,9,10]$. For a comprehensive review of past work see [11]. 
In this paper we consider a simple and easily computed statistic for tree shape -namely the number of pairs of leaves that are adjacent to a common ancestor node - such a pair of leaves we will call a cherry. We will analyse the distribution of this statistic under the two simplest models for generating phylogenetic trees, namely the Yule model, and the uniform model. In particular, we give exact formulae for the mean and variance under both distributions, and show that this distribution is asymptotically normal as the number of leaves grows.

The structure of this paper is as follows. Section 2 reviews some basic terminology for phylogenetic binary trees. Following this, extended Polya urn models are described in Section 3. In Section 4 the Yule and uniform models are explained, and asymptotic normality results for the number of cherries is derived, along with exact formulae for the mean and variance. These results are used to develop statistical test for the Yule and uniform null hypotheses. The power of these tests is also calculated, using the other model as the alternative hypothesis. Section 5 concludes with a brief application to a 34 -species tree.

\section{Terminology}

Evolutionary relationships are often represented by rooted or unrooted binary (phylogenetic) trees [12]. Such trees consist of labeled nodes of degree 1 called leaves and unlabelled internal nodes of degree 3 (also, in case the tree is rooted, it contains an additional root node of degree 2 , so that every node can be regarded as having exactly two descendants). A pair of leaves adjacent to a common node is called a cherry. Edges adjacent to a leaf are called pendant edges, while all other edges are internal. A (tree) shape is the unlabeled tree obtained by dropping the labeling of the leaves of a binary phylogenetic tree. For further clarification of these terms see Figure 1. The number of rooted leaf-labeled binary trees on $n$ leaves is given by $(2 n-3) ! !=1 \times 3 \times 5 \cdots \times(2 n-3)$, and the number of unrooted leaf-labeled binary trees on $n$ leaves is given by $(2 n-5) ! !=1 \times 3 \times 5 \cdots \times(2 n-5)$.

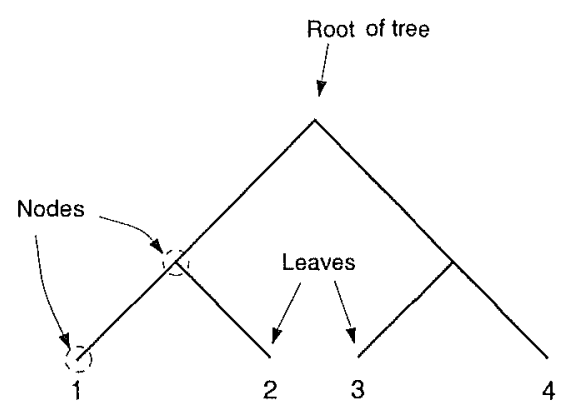

(i)

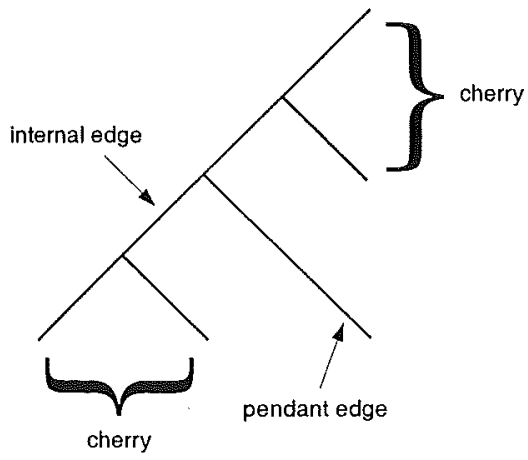

(ii)

Figure 1: (i) A labeled rooted tree with 4 leaves. (ii) An unrooted tree shape with 5 leaves. 


\section{Extended Polya Urn (EPU) Models}

In this section we review a recent central limit theorem concerning a general type of urn model, which will be useful for describing the asymptotic distribution of cherries.

Suppose an urn contains $p$ types of balls. If a ball of the $i-t h$ type $(i \in\{1, \ldots, p\})$ is drawn from the urn then it is returned, along with $A_{i j}$ balls of the $j$-th type. $A_{i j}$ can be negative, this corresponding to the removal of balls from the urn. Models with $A_{i i} \geq 0$ (and commonly $A_{i j} \geq 0$ ) are referred to as generalized polya urn (GPU) models $[13,14]$. Allowing for $A_{i j}$ to be negative, but requiring the number of balls returned each time to be a constant, defines the class of extended polya urn (EPU) models $[15,16]$.

For both classes of urn models a number of asymptotic normal distributions results exist, but in this paper we need only consider some specific asymptotic results for the EPU model, as follows $[15,16]$.

Theorem 1 Let $A=\left[A_{i j}\right]$ be the generating matrix for an EPU model, with principal eigenvalue $\lambda_{1}$. Let $v$ be the left eigenvector of $A$ corresponding to $\lambda_{1}$, where the entries $v_{i}$ add up to one. Also let $Z_{\text {in }}$ denote the number of balls of type $i$ in the urn after $n$ draus, where $i=1,2, \ldots$, p. For $p=2$ suppose that:

(i) A has constant row sums,

(ii) $A^{2}$ is finite,

(iii) $\lambda_{1}$ is positive, simple, and has a strictly positive left eigenvector $v$,

(iv) $2 \lambda \leq \lambda_{1}$ for all non-principal eigenvalues $\lambda$;

then $n^{-1 / 2}\left(Z_{1 n}-n \lambda_{1} v_{1}\right)$ has asymptotically a normal distribution with mean of zero.

Furthermore, for $p>2$, suppose in addition:

(v) $2 \operatorname{Re}(\lambda)<\lambda_{1}$ for all non-principal eigenvalues $\lambda$,

(vi) all complex eigenvalues are simple,

(vii) all eigenvectors are linearly independent;

then $n^{-1 / 2}\left(Z_{1 n}-n \lambda_{1} v_{1}, Z_{2 n}-n \lambda_{1} v_{2}, \ldots, Z_{(p-1) n}-n \lambda_{1} v_{(p-1)}\right)$ has asymptotically a jount normal distribution with mean of zero.

\section{Probability Distribution For Cherries}

\subsection{Yule Model}

In the Yule model on rooted trees each pendant edge has an equal probability of splitting to form a cherry [17]. Equivalently an edge is added uniformly and randomly to a pendant edge at each step (Figure 2). This model assumes that speciation is instantaneous, always 
occurs as bifurcations, and that the probability of speciation is the same for all lineages at any given time [18]. Extinction may be incorporated into this model by assuming that the probability of extinction is the same for all lineages, and independent across lineages. If this is the case then a Yule model can still be used, but with a different 'speciation' rate $[5,19]$.

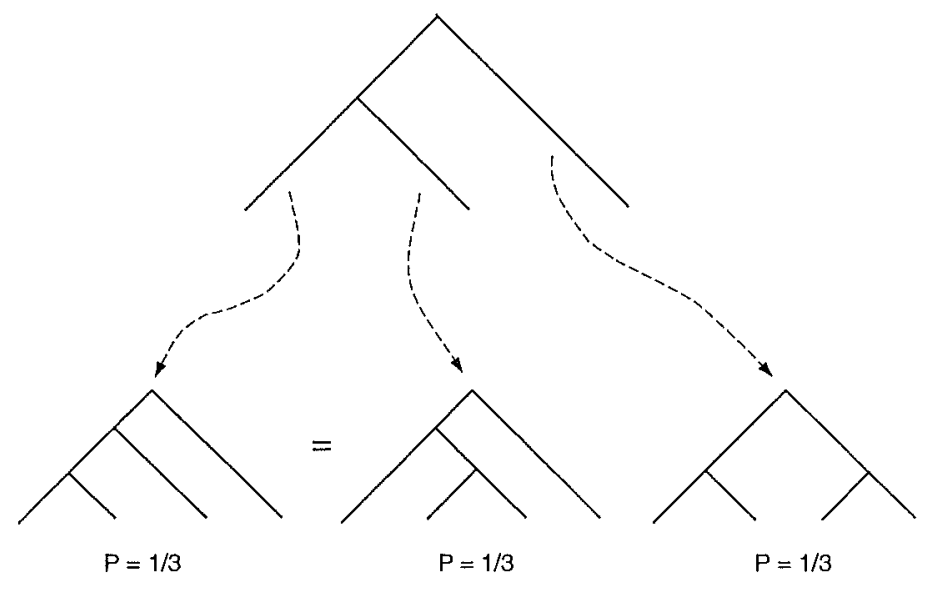

Figure 2: The Yule model probabilities for shapes with 4 leaves. A shape on 4 leaves is formed by the splitting of one the pendant edges of the shape on 3 leaves. Each pendant edge has the same probability of splitting, so for the shape on 3 leaves each pendant edge has a probability of $1 / 3$ of splitting. The resulting symmetric shape on 4 leaves has a probability of $1 / 3$. The other two shapes on 4 leaves are the same, and so the probability of this shape is $2 / 3$.

Theorem 2 Let $\mu_{n}$ be the mean number of cherries for a rooted binary tree on $n$ leaves, and $\sigma_{n}^{2}$ be the variance for the number of cherries. Under the Yule distribution we have the recursions, for $n \geq 2$ :

$$
\mu_{n+1}=1+\mu_{n}\left(1-\frac{2}{n}\right) ; \quad \sigma_{n+1}^{2}=\sigma_{n}^{2}\left(1-\frac{4}{n}\right)+\frac{2}{n} \mu_{n}\left(1-\frac{2}{n} \mu_{n}\right)
$$

which may be solved exactly to give

$$
\mu_{n}=\frac{n}{3} \quad(n \geq 3) ; \quad \sigma_{n}^{2}=\frac{2 n}{45} \quad(n \geq 5) .
$$

Proof. Let random variable $C_{n}$ be the number of cherries on a rooted binary tree on $n$ leaves. The proof relies on the following recursion for the probability generating function for $C_{n}$ under the Yule model. Let $P_{n}(x):=\sum_{k \geq 2} \mathbb{P}\left[C_{n}=k\right] x^{k}$. Then, from Steel and Penny [20], we have the recursion, for $n \geq 4$ :

$$
P_{n+1}(x)=x P_{n}(x)+\frac{2 x}{n}(1-x) \frac{d}{d x} P_{n}(x)
$$


and from this the recursions for the mean and variance follow directly by the usual techniques (noting that $\mu_{n}=\left.\frac{d}{d x} P_{n}(x)\right|_{x=1}$ and $\sigma_{n}^{2}=\left.\frac{d^{2}}{d x^{2}} P_{n}(x)\right|_{x=1}+\mu_{n}-\mu_{n}^{2}$ ). The stated explicit formulas for the mean and variance can then be verified by induction on $n$.

The asymptotic probability distribution for the number of cherries in the Yule model may be found by realizing the process of cherry formation in an EPU model. Let the pendant edges that are part of a cherry be represented by black balls, and the rest of the pendant edges be represented by white balls. The number of cherries is then half the number of black balls, and the total number of pendant edges is equal to the number of black and white balls.

The following urn scheme generates a probability distribution for the number of black balls that is equal to the probability distribution for the number of pendant edges that are part of cherries. Starting with a rooted binary tree with two leaves, put two black balls into an empty urn. Select a ball at random from the urn, then return it. If the ball selected was black then put in a white ball. If the ball selected was white then put in two black balls, and take out a white ball. Repeat the process of random selection, and the addition or removal of balls $n-2$ times, until there are $n$ balls in the urn.

The generating matrix for this urn scheme is

$$
A=\left(\begin{array}{rr}
0 & 1 \\
2 & -1
\end{array}\right)
$$

where balls of type one are black, and those of type two are white.

The eigenvalues of $A$ are the principal eigenvalue $\lambda_{1}=1$, and $\lambda_{2}=-2$. For the principal eigenvalue the left eigenvector, for which the entries add up to one, is $v=(2 / 31 / 3)^{T}$. The conditions for the EPU model asymptotic results to apply in Theorem 1 are clearly satisfied so

$$
\frac{1}{\sqrt{n}}\left(Z_{1 n}-2 n / 3\right) \rightarrow \mathcal{N}(0, c)
$$

where $\mathcal{N}\left(\mu, \sigma^{2}\right)$ is a normal distribution with mean $\mu$ and variance $\sigma^{2}$. Substituting $Z_{1 n}=2 C_{n}$ gives

$$
C_{n} \rightarrow \mathcal{N}(n / 3, c n / 2)
$$

Using Theorem 2 the value of the constant $c$ can be identified giving the following result.

Theorem 3 For the Yule model on rooted trees

$$
C_{n} \rightarrow \mathcal{N}(n / 3,2 n / 45)
$$

\subsection{Uniform Model}

In the uniform model on unrooted trees equal probability is assigned to each possible leaf-labeled binary tree on $n$ leaves. Alternatively, in the uniform model an edge is added uniformly and randomly to any edge at each step (Figure 3). This process is not an explicit 
model of evolution, though a random sample of $n$ species from a large group of species generated by a conditioned branching process follows a uniform distribution [21, 22].

Theorem 4 Let $\mu_{n}$ be the mean for $C_{n}$ for an unrooted binary tree on $n$ leaves, and $\sigma_{n}^{2}$ be the variance for $C_{n}$. Under the uniform model, for $n \geq 4$,

(a)

$$
\mathbb{P}\left[C_{n}=k\right]=\frac{n !(n-2) !(n-4) ! 2^{n-2 k}}{(n-2 k) !(2 n-4) ! k !(k-2) !}, \quad k \geq 2
$$

$$
\mu_{n}=\frac{n(n-1)}{2(2 n-5)} \sim \frac{n}{4} ; \quad \sigma_{n}^{2}=\frac{n(n-1)(n-4)(n-5)}{2(2 n-5)^{2}(2 n-7)} \sim \frac{n}{16}
$$

Proof. Part (a) is due to Hendy and Penny [23], while the first part of (b) appears in Steel and Penny [20]. For the second (variance) part of (b) we note from Steel [24] that the $p$ - th cumulative moment of $C_{n}$ is $\frac{(2(n-s)-5) ! ! n !}{2^{s}(2 n-5) ! !(n-2 s) !}$ (where the !! notation is as in Section 2) and thus,

$$
\sigma_{n}^{2}=\frac{n(n-1)(n-2)(n-3)}{2^{2}(2 n-5)(2 n-7)}+\mu_{n}-\mu_{n}^{2}
$$

Rearranging this last equation gives the result.

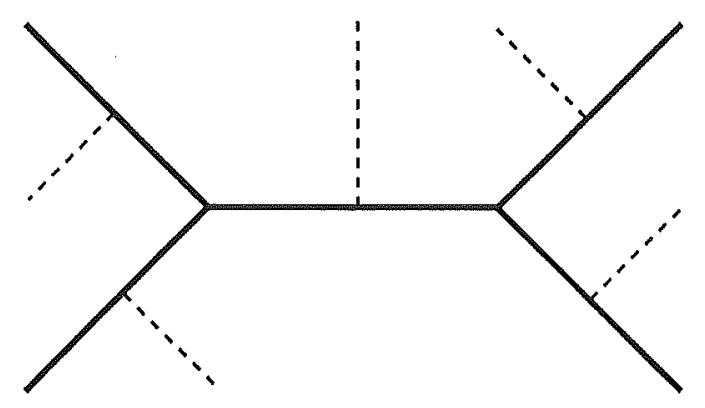

Figure 3: The uniform model on unrooted trees. For the unrooted shape on 4 leaves (suliul lines) there are five possible edges where the next edge (dashed lines) may be attached. The probability that the next edge will be attached to any particular one of the possille. edges is $1 / 5$.

The asymptotic probability distribution for the number of cherries in the uniform morle! may be found by an extension of the urn scheme for the Yule model. As before, let pendinu edges that are part of cherries be represented by black balls and the other pendant edges by white balls. In addition, let the internal edges be represented by red balls.

The uniform model, with regard to the number of cherries, is equivalent to the following urn scheme. Starting with an unrooted binary tree with four leaves, put four black balls 
and one red ball into an empty urn. Select a ball at random from the urn, then return it. If the ball selected was black then put in a white ball and a red ball. If the ball selected was white then put in two black balls, take out a white ball, and put in a red ball. If the ball selected was red then put in a white ball and a red ball. Repeat the process of random selection, and the addition or removal of balls $n-4$ times, until there are $n$ balls in the urn.

The generating matrix for this urn scheme is

$$
A=\left(\begin{array}{rrr}
0 & 1 & 1 \\
2 & -1 & 1 \\
0 & 1 & 1
\end{array}\right)
$$

where balls of type one are black, type two are white, and type three are red.

The eigenvalues of $A$ are $-2,0$ and the principal eigenvalue of $\lambda_{1}=2$. For the principal eigenvalue the left eigenvector of $A$, for which the entries add up to one, is v $=(1 / 41 / 41 / 2)^{T}$. The conditions for the EPU model asymptotic results to apply in Theorem 1 are clearly satisfied so

$$
\frac{1}{\sqrt{n}}\left(Z_{1 n}-n / 2\right) \rightarrow \mathcal{N}(0, c)
$$

Substituting $Z_{1 n}=2 C_{n}$ gives

$$
C_{n} \rightarrow \mathcal{N}(n / 4, c n / 4)
$$

Using Theorem 4 the value of the constant $c$ can be identified, giving the following result.

Theorem 5 For the uniform model on unrooted trees

$$
C_{n} \rightarrow \mathcal{N}(n / 4, n / 16)
$$

\subsection{Rooted and Unrooted Trees}

The Yule and uniform models, as stochastic processes involving random edge addition, can apply to both rooted and unrooted trees. In the Yule model an edge is added uniformly and randomly to a pendant edge, while in the uniform model an edge is added uniformly and randomly to any edge (allowing a 'ghost' edge at the root in the rooted case). For the process of generating leaf-labeled trees two possible schemes are to add taxa in either fixed order or uniformly randomly. For the Yule model the taxa must be added uniformly randomly in order to generate the correct probability distribution on leaf-labeled trees, while for the uniform model either scheme can be used.

We have so far only considered the cherry distribution for the Yule process on rooted trees and the uniform process on unrooted trees. How does the cherry distribution change if rooted trees are "unrooted" by suppressing the root, or if unrooted trees are "rooted" by the introduction of a root? A related question is what is the cherry distribution for the Yule process on unrooted trees, and the uniform process on rooted trees?

Consider firstly the unrooting of a rooted tree. Let $T$ be a rooted tree on $n$ leaves, with the number of cherries given by the random variable $C_{n}$. If the (degree 2 ) root of $T$ is 
suppressed then the number of cherries $\left(C_{n}^{*}\right)$ either remains the same, or increases by one; the latter occurs precisely when the tree shape has the generic shape shown in Figure 4. Let $D_{n}=C_{n}^{*}-C_{n} \in\{0,1\}$.

\section{Lemma 1}

(a) For the Yule model on rooted trees

$$
\lim _{n \rightarrow \infty} \mathbb{P}\left[D_{n}=1\right]=0
$$

(b) For the uniform model on rooted trees

$$
\lim _{n \rightarrow \infty} \mathbb{P}\left[D_{n}=1\right]=1 / 4
$$

Proof. $D_{n}$ equals one precisely when the rooted tree shape is as shown in Figure 4. For the Yule model, two applications of a recursive formula for tree probabilities [17] gives

$$
\mathbb{P}\left[D_{n}=1\right]=\frac{4}{(n-1)(n-2)} \sum_{\tau} \mathbb{P}[\tau]=\frac{4}{(n-1)(n-2)},
$$

where the summation is over all subtree shapes $\tau$ on $n-2$ leaves. Taking the limit gives the required result. For part (b), there are $(2 n-3)$ !! possible leaf-labeled rooted trees on $n$ leaves. Of these $n(n-1)(2 n-7)$ !! have the shape shown in Figure 4 . Therefore

$$
\mathbb{P}\left[D_{n}=1\right]=\frac{n(n-1)(2 n-7) !}{(2 n-3) ! !}=\frac{n(n-1)}{(2 n-3)(2 n-5)},
$$

and taking the limit gives the required result for the uniform model.

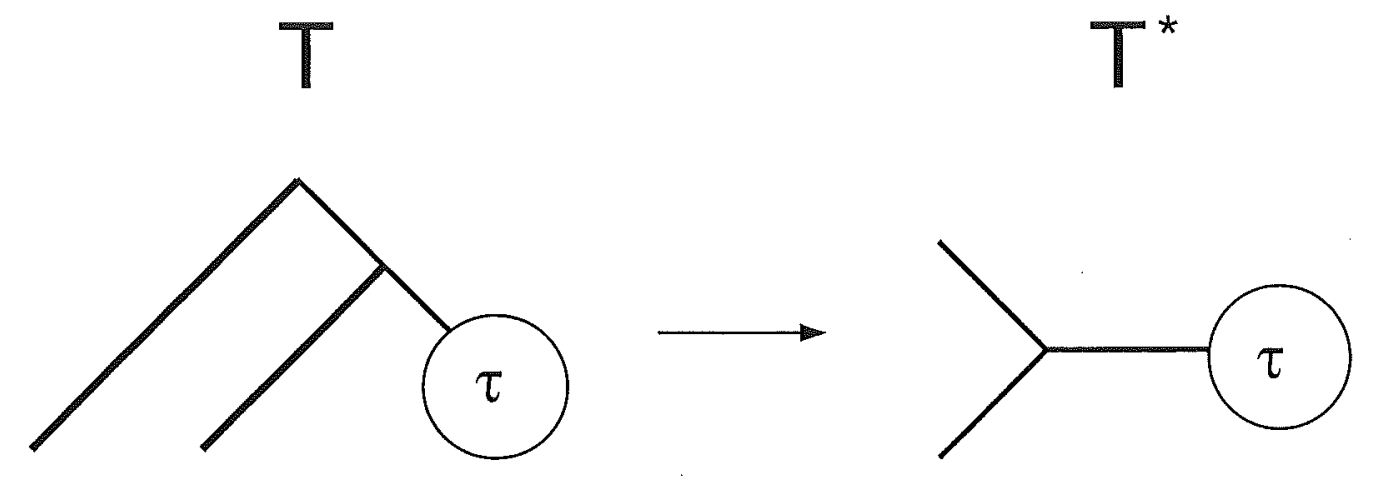

Figure 4: Rooted binary tree $T$ for which the associated unrooted tree $T^{*}$ has one more cherry. The tree $T$ has $n$ leaves, and the subtree $\tau$ has $n-2$ leaves.

If an unrooted tree is rooted, by subdividing some edge, then the number of cherries either remains the same or decreases by one; the latter occurs precisely when the tree is rooted 
on an edge that is part of a cherry. Since rooting or unrooting a tree changes the number of cherries by a maximum of one the asymptotic probability distribution for the number of cherries will remain unchanged.

Furthermore, the Yule and uniform processes can apply on both rooted and unrooted trees. For both processes the generating matrix for the corresponding EPU model is the same in the rooted and unrooted cases. Therefore, the asymptotic probability distribution for the number of cherries is the same for the rooted and unrooted versions of the Yule and uniform processes.

\subsection{The Yule model null hypothesis}

The Yule model can be used as a simple null hypothesis to explore patterns in phylogenetic trees. A simple two-tailed test of the Yule null hypothesis, for a given tree, can be made based on the number of cherries in the tree. If the number of cherries is below some lower critical value, or above some upper critical value, then the Yule null hypothesis is rejected. For small $n$, a recursive formula for the probabilities implicit in equation (1) may be used to calculate the rejection limits (Figure 6). For larger values of $n(n \gtrsim 20)$ a normal approximation is valid. In this case, based on Theorem 3 , the rejection region for a twosided test at the $\alpha$ level is given by

$$
C_{n}<\frac{n}{3}-Z_{\frac{\alpha}{2}} \sqrt{\frac{2 n}{45}} \quad \text { and } \quad C_{n}>\frac{n}{3}+Z_{\frac{\alpha}{2}} \sqrt{\frac{2 n}{45}}
$$

The lower and upper critical values for rejection at an $\alpha=0.05$ level are shown in Figure 7. If the Yule model is rejected then this implies that one or more of the assumptions upon which it is based is invalid. Often it is assumed that the assumption of equal probability of speciation is the invalid assumption, but this need not be the case [18].

\subsection{Uniform model null hypothesis}

In the uniform model equal probability is assigned to each possible leaf-labeled binary tree on $n$ leaves. Thus the uniform model distribution may be used to model the frequency of outcomes that would occur if the process of tree reconstruction did no better than random selection from the set of possible trees on $n$ leaves. A test of the uniform model null hypothesis may be constructed based on the number of cherries in a tree. For small $n$ the probability distribution given in Theorem 4 may be used to calculate the rejection limits (Figure 6$)$. For larger $n(n \gtrsim 20)$ a analysis similar to that for the Yule model, but based on Theorem 5 , gives as the rejection region:

$$
C_{n}<\frac{n}{4}-Z_{\frac{\alpha}{2}} \sqrt{\frac{n}{16}} \quad \text { and } \quad C_{n}>\frac{n}{4}+Z_{\frac{\alpha}{2}} \sqrt{\frac{n}{16}} .
$$

The lower and upper critical values for rejection at an $\alpha=0.05$ level are shown in Figure 7 .

\subsection{Power of Tests}

The power of a test is the probability that the null hypothesis will be rejected given that the alternative hypothesis is true. Calculating the power of the test for the Yule null 
hypothesis against the uniform model alternative hypothesis gives

$$
\operatorname{power}(n)=\mathbb{P}\left[Z<\sqrt{n} / 3-r_{1}\right]+\mathbb{P}\left[Z>\sqrt{n} / 3+r_{1}\right], \quad r_{1}=4 \sqrt{\frac{2}{45}} Z_{\frac{\alpha}{2}} .
$$

Similarly, the power of the test for the uniform model null hypothesis may be calculated against the alternative hypothesis that the Yule model is true to give

$$
\operatorname{power}(n)=\mathbb{P}\left[Z<-\frac{1}{4} \sqrt{\frac{5}{2}} \sqrt{n}-r_{2}\right]+\mathbb{P}\left[Z>-\frac{1}{4} \sqrt{\frac{5}{2}} \sqrt{n}+r_{2}\right], \quad r_{2}=\frac{1}{4} \sqrt{\frac{45}{2}} Z_{\frac{\alpha}{2}} .
$$

Plotting the power, as a function of $n$, shows that in both cases the number of leaves must exceed 80 before the power of the tests rises above 0.9 (Figure 5). So, unless one is dealing with large trees, the tests lacks the power to distinguish between the two models.

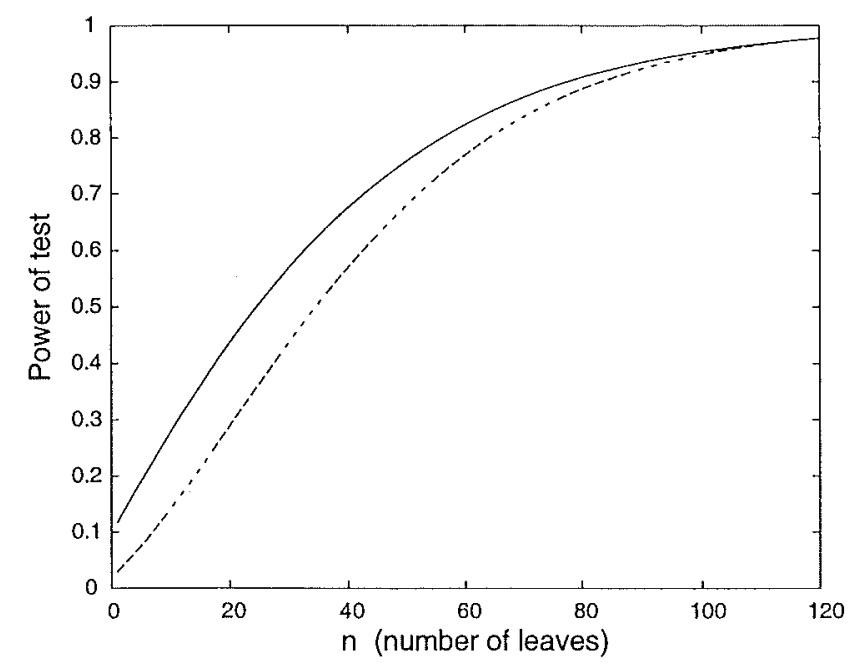

Figure 5: The power of the tests for the Yule and uniform models. The solid line is the power of the test for the Yule model null hypothesis against the uniform model alternative. The dashed line is the power of the test for the uniform model null hypothesis test against the Yule model alternative.

\section{An Example}

Figure 1 in [25] is a rooted phylogenetic tree for 34 species of eureptantic nemerteans (ribbon worms). This tree has 7 cherries (rooted or unrooted). For the Yule model null hypothesis test at the $\alpha=0.05$ level the lower rejection limit is 8 cherries or less, and the upper rejection limit is 15 cherries or more. So for the ribbon worm tree the Yule model null hypothesis is rejected. For the uniform model null hypothesis test at the $\alpha=0.05$ level the lower rejection limit is 5 cherries or less, and the upper rejection limit is 13 cherries or more, and so the test does not reject the uniform model null hypothesis. 


\subsection{Acknowledgement}

We thank Charles Semple for some helpful comments concerning an earlier version of this manuscript. 


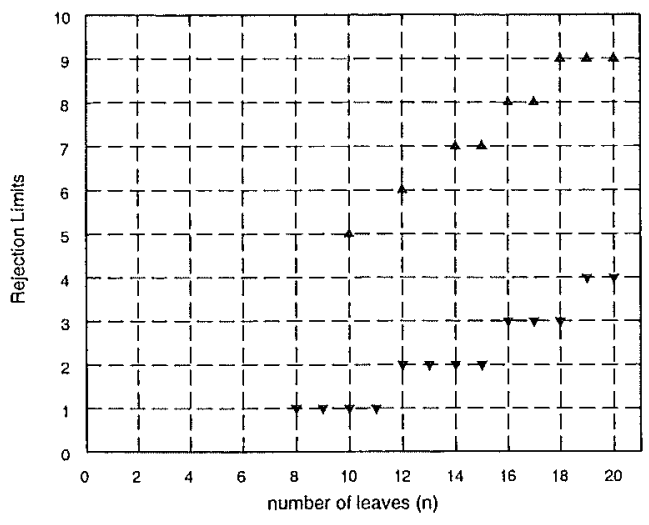

(i) Yule model

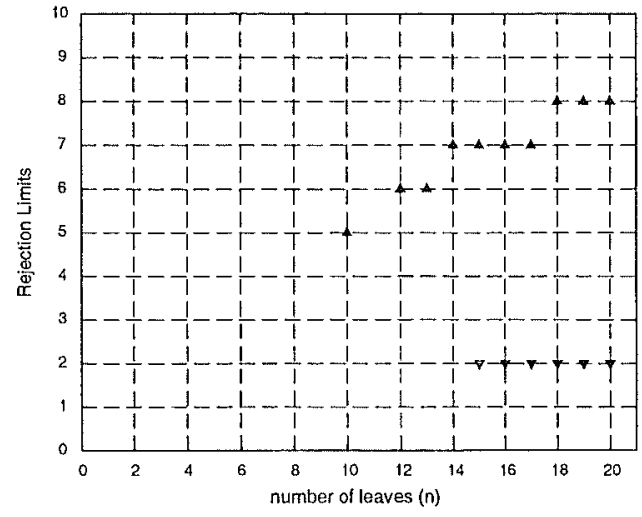

(ii) Uniform model

Figure 6: Rejection limits for small $n$ of the Yule and uniform null hypotheses at the $\alpha=$ 0.05 level. Lower limits $(\mathbf{v})$ and upper limits $(\mathbf{\Delta})$ were calculated from the exact probability distribution for the number of cherries. Where no triangle is shown the rejection limit does not exist.

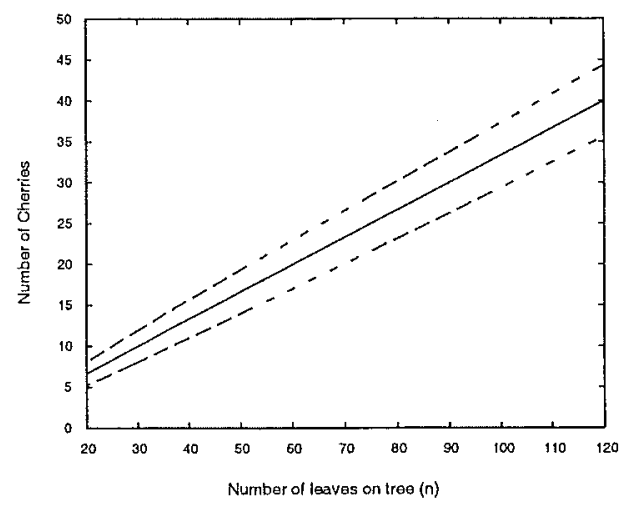

(i) Yule model

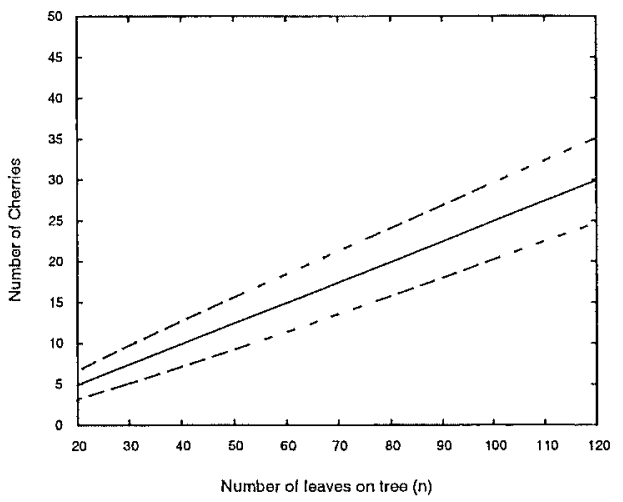

(ii) Uniform model

Figure 7: Rejection limits for large $n$ of the Yule and uniform null hypotheses at the $\alpha=0.05$ level. The solid line represents the mean number of cherries, while the dashed lines are the lower and upper limits for rejection of the null hypotheses. The rejection limits are based upon a normal approximation which is valid for $\mathrm{n} \gtrsim 20$. 


\section{References}

[1] T. Kubo and Y. Iwasa. Inferring the rates of branching and extinction from molecular phylogenies. Evolution, 49:694-704, 1995.

[2] D. M. Raup, S. J. Gould, T. J. M. Schopf, and Daniel S. Simberloff. Stochastic models of phylogeny and the evolution of diversity. The Journal of Geology, 81(5):525-542, Sep 1973.

[3] S. J. Gould, D. M. Raup, J. J. Sepkowski, T. J. M. Schopf, and D. S. Simberloff. The shape of evolution: a comparison of real and random clades. Paleobiology, 3:23-40, 1977 .

[4] H. M. Savage. The shape of evolution: systematic tree topology. Biological Journal of the Linnean Society, 20:225-244, 1983.

[5] J. B. Slowinski and C. Guyer. Testing the stochasticity of patterns of organismal diversity: an improved null model. The American Naturalist, 134(6):907-921, Dec 1989 .

[6] C. Guyer and J. B. Slowinski. Comparisons of observed phylogenetic topologies with null expectations among three monophyletic lineages. Evolution, 45(2):340-350, 1991.

[7] S. B. Heard. Patterns in tree balance among cladistic, phenetic, and randomly generated phylogenetic trees. Evolution, 46(6):1818-1826, 1992.

[8] M. Kirkpatrick and M. Slatkin. Searching for evolutionary patterns in the shape of a phylogenetic tree. Evolution, 47(4):1171-1181, 1993.

[9] J. S. Rogers. Central moments and probability distribution of Colless's coefficient of tree imbalance. Evolution, 48(6):2026-2036, 1994.

[10] A. Mooers. Tree balance and tree completeness. Evolution, 49(2):379-384, 1995.

[11] A. Mooers and S. B. Heard. Inferring evolutionary process from phylogenetic tree shape. The Quarterly Review of Biology, 72(1):31-54, Mar 1997.

[12] R. D. M. Page and E. C. Holmes. Molecular Evolution: a phylogenetic approach, chapter 2, pages 11-36. Blackwell Science, 1998.

[13] K. B. Athreya and S. Karlin. Embedding of urn schemes into continuous time markov branching processes and related limit theorems. Ann. Math. Statist., 39:1801-1817, 1968 .

[14] K. B. Athreya and P. E. Ney. Branching Processes, pages 219-224. Springer-Verlang, 1972.

[15] A. Bagchi and A. K. Pal. Asymptotic normality in the generalized Polya-Eggenburger urn model, with an application to computer data structures. SIAM J. Algebraic Discrete Methods, 6:394-405, 1985. 
[16] R. T. Smythe. Central limit theorems for urn models. Stochastic Processes and their Applications, 65:115-137, 1996.

[17] E. F. Harding. The probabilities of rooted tree-shapes generated by random bifurcation. Adv. Appl. Prob., 3:44-77, 1971.

[18] J. B. Losos and F. R. Adler. Stumped by trees? a generalized null model for patterns of organismal diversity. The American Naturalist, 145(3):329-342, Mar 1995.

[19] S. Nee, R. M. May, and P. H. Harvey. The reconstructed evolutionary process. Phil. Trans. R. Soc. Lond. B, 344:305-311, 1994.

[20] M. Steel and D. Penny. Distribution of tree comparison metrics - some new results. Syst. Biol., 42(2):126-141, 1993.

[21] D. Aldous. The continuum random tree II: an overview. In M. T. Barlow and N. H. Bingham, editors, Stochastic Analysis, pages 23-70. Cambridge University Press, Cambridge, 1991.

[22] D. Aldous. The continuum random tree III. Ann. Probab., 21:248-289, 1993.

[23] M. D. Hendy and D. Penny. Branch and bound algorithms to determine minimal evolutionary trees. Math. Biosci., 59:277-290, 1982.

[24] M. A. Steel. Distribution of the symmetric difference metric on phylogenetic trees. SIAM J. Discr. Math., 1(4):541-551, 1988.

[25] M. Härlin. Biogeographic patterns and the evolution of eureptanic nemerteans. Biological Journal of the Linnean Society, 58:325-342, 1996. 